

\title{
Semi-parametric estimation of joint large movements of risky assets
}

\author{
Alexandra Dias* \\ Finance Group \\ Warwick Business School \\ University of Warwick
}

August 9, 2008

\begin{abstract}
The classical approach to modelling the occurrence of joint large movements of asset returns is to assume multivariate normality for the distribution of asset returns. This implies independence between large returns. However, it is now recognised by both academics and practitioners that large movements of assets returns do not occur independently. This fact encourages the modelling joint large movements of asset returns as non-normal, a non trivial task mainly due to the natural scarcity of such extreme events.

This paper shows how to estimate the probability of joint large movements of asset prices using a semi-parametric approach borrowed from extreme value theory (EVT). It helps to understand the contribution of individual assets to large portfolio losses in terms of joint large movements. The advantages of this approach are that it does not require the assumption of a specific parametric form for the dependence structure of the joint large movements, avoiding the model misspecification; it addresses specifically the scarcity of data which is a problem for the reliable fitting of fully parametric models; and it is applicable to portfolios of many assets: there is no dimension explosion.

The paper includes an empirical analysis of international equity data showing how to implement semi-parametric EVT modelling and how to exploit its strengths to help understand the probability of joint large movements. We estimate the probability of joint large losses in a portfolio composed of the FTSE 100, Nikkei 250 and S\&P 500 indices. Each of the index returns is found to be heavy tailed. The S\&P 500 index has a much stronger effect on large portfolio losses than the FTSE 100, although having similar univariate tail heaviness.
\end{abstract}

\section{Introduction}

Modelling the existence of joint large movements of asset prices in financial theory may potentially lead to significant improvements in specific areas of finance, such as asset pricing, optimal

\footnotetext{
* Correspondence address: Alexandra Dias, Warwick Business School, Finance Group, University of Warwick, CV4 7AL Coventry, UK. E-mail: Alexandra.Dias@wbs.ac.uk.

I am grateful for comments on earlier versions from Nick Webber and seminar participants at Warwick Business School, Bank of England and Bachelier Finance Society Conference 2008.
} 
portfolio choice, derivatives valuation and hedging, and management and measurement of financial risks. However, the construction and estimation of models taking into account large movements of asset prices is a non-trivial task. Large movements may be directly modelled but more often their characteristics are implicitly assumed by adopting a general probability distribution used to model the asset prices. There is no economic or statistical theory supporting any specific probability distribution for joint large asset returns. Hence, a model has to be assumed which may or may not be based on data analysis. For decades the standard probability distribution selected for asset prices was the multivariate normal. Still today, financial regulators base their directives largely on models with an underlying normal distribution.

Assuming a multivariate normal distribution for asset price returns implies that, asymptotically, large joint price movements occur independently. Yet, financial market crashes, for instance those occurred in 1929 or in 1987, had effects across several markets and financial institutions. Hence the multivariate normal distribution may underestimate the probability of joint large financial events. The need for alternative models has been recognised by practitioners and academics.

Attempts to depart from normality have been made specifically in terms of modelling large events. Examples of studies focussed on the univariate behavior of large movements in financial markets are Cotter (2006), Danielsson and de Vries (2000), Jansen and de Vries (1991), Longin (1996), Longin (2005) and McNeil and Frey (2000). There is not much literature on the more difficult case of modelling multivariate large movements: Longin and Solnik (2001) study the extreme correlation between international markets by modelling the dependence of bivariate extreme events with the logistic function proposed by Gumbel (1961). Poon et al. (2004) emphasize the importance of distinguishing between dependent and independent extreme events and use the logistic function to model the case of dependence.

In these studies, the dependence structure between the extreme events in different markets is estimated through a parametric model from extreme value theory assumed a priori. The natural scarcity of observed extreme events does not facilitate model specification tests. A related reference on the pitfalls and opportunities in the use of extreme value theory in finance is Diebold et al. (1998). Further, when the number of risk factors increases the appropriateness of a parametric model becomes dubious, its estimation more difficult and the results less reliable.

In this paper we show how to estimate the probability of joint large movements of asset 
prices using a semi-parametric method from extreme value theory. To our knowledge this has not been used in finance applications before. This method was originally developed to model extreme weather conditions in the North Sea; see de Haan and de Ronde (1998). The approach is semi-parametric because no specific form for the dependence structure of the extreme movements is assumed. This reduces the problem of model misspecification and increases the significance of the results compared with fully parametric models. Another advantage of the methodology is that the estimation of the probability of joint extreme price movements does not become significantly more difficult as the number of assets increases.

We illustrate the method with an empirical study where we explore the extremal dependence structure among three major international stock indexes: FTSE 100, Nikkei 225 and S\&P 500. We obtain estimates for the probability of occurrence of very large losses in a portfolio composed of the three indices. We exploit the potentialities of the methodology by studying the effect of each index on the probability of large portfolio losses.

This paper is organized as follows. The first section presents the theoretical results we use from extreme value theory. The second section deals with the methods of statistical estimation. Section 3 contains the empirical analysis and the fourth section concludes.

\section{Joint large movements: theory}

Very large joint movements of asset prices do not occur frequently and this is why extreme value theory is called for. Extreme value theory provides a model for the multivariate distribution of the maximum return ${ }^{1}$ observed for each asset over a period of time. We denote by $\mathbf{R}=$ $\left(R_{1}, R_{2}, \ldots, R_{d}\right)$ the random vector representing the one period loss returns of $d$ assets. A main result in extreme value theory is that for the distributions commonly used for $\mathbf{R}$ there is a limit distribution for their componentwise maxima. In the following we explain this and consequent results useful for the problem addressed in this paper.

Let $\mathbf{R}_{i}, i=1,2, \ldots, n$ denote the vector of returns in period $i$. If the random vectors $\mathbf{R}_{1}, \mathbf{R}_{2}, \ldots, \mathbf{R}_{n}$ are independent and identically distributed, drawn from a distribution $F_{\mathbf{R}}$, then the distribution of the componentwise maxima is the power $F_{\mathbf{R}}^{n}(\mathbf{x})$. This distribution is usually unknown. To overcome this, extreme value theory derives the limit distribution of

\footnotetext{
${ }^{1}$ Often we are interested in modelling the minimum return but this is equivalent to modelling the maximum loss return. Hence the same results can be used for the minimum return.
} 
$F_{\mathbf{R}}^{n}(\mathbf{x})$ when $n$ goes to infinity, resembling the approach used for deriving the Central Limit Theorem (CLT). Like the CLT case, the componentwise maxima have to be standardized in order to have a non-degenerate limit distribution. We denote by $\overline{\mathbf{R}}_{n}$ the vector of standardized componentwise maxima, so that,

$$
\overline{\mathbf{R}}_{n}=\mathbf{a}^{-1}\left(\max \left(\mathbf{R}_{1}, \mathbf{R}_{2}, \ldots, \mathbf{R}_{n}\right)-\mathbf{b}\right)
$$

where $\mathbf{a}$ and $\mathbf{b}$ are vectors of scale and location coefficients depending on $n .^{2}$

From extreme value theory (see de Haan and Resnick (1977)) there is a non-degenerate limit distribution $G(\mathbf{x})$ for the normalized maxima, the so called extreme value distribution, such that

$$
\lim _{n \rightarrow \infty} P\left(\overline{\mathbf{R}}_{n} \leq \mathbf{x}\right)=G(\mathbf{x})
$$

This implies convergence for the marginal distributions, in particular, that each univariate marginal $G_{j}(x), j=1, \ldots, d$ is an extreme value distribution. In fact, there are normalizing constants $a_{j}>0$ and $b_{j} \in \mathbb{R}$ such that each univariate marginal distribution is of the form

$$
G_{j}(x)=\exp \left(-\left(1+\gamma_{j} \frac{x-b_{j}}{a_{j}}\right)^{-1 / \gamma_{j}}\right),
$$

for $1+\gamma_{j}\left(x-b_{j}\right) / a_{j}>0$ and where $\gamma_{j} \in \mathbb{R}$ is a shape parameter.

Let $G$ be a univariate extreme value distribution. If $\gamma<0$ then $G$ is a Weibull distribution, if $\gamma=0$ then $G$ is a Gumbel distribution and if $\gamma>0$ then $G$ is a (heavy tailed) Fréchet distribution (see Embrechts et al. (1997)). In the last case where $\gamma>0$ the shape parameter $\gamma$ is known as the tail index.

A model for the distribution of the vector of returns $\mathbf{R}$ allows us to estimate the probability of events which depend on a function $f(\mathbf{R})$ of these returns. An example is the estimation of the probability of a large portfolio loss return. In this case, the function $f: \mathbb{R}^{d} \rightarrow \mathbb{R}$ transforms the individual asset returns into a portfolio loss return. For example if we want to estimate the probability that the portfolio loss return is larger than a given threshold $L, L=20 \%$ say, this means, in terms of our notation, estimating $P(f(\mathbf{R})>L)$.

Given an event depending on a function $f(\mathbf{R})$ of the asset returns, there is a set $C \subseteq$ $\mathbb{R}^{d}$ of joint returns satisfying the condition defining the event whose probability we want to

\footnotetext{
${ }^{2}$ Here and elsewhere operations (maximum, addition, multiplication and taking powers) are componentwise.
} 
estimate. For the case of a portfolio loss larger than $L$ this means that estimating the probability $P(f(\mathbf{R})>L)$ is equivalent to estimating $P(\mathbf{R} \in C)$ where $C$ contains all the possible large joint loss returns $\mathbf{R}$ such that $f(\mathbf{R})>L, C=\left\{\mathbf{R} \in \mathbb{R}^{d}: f(\mathbf{R})>L\right\}$.

For loss values $L$ of interest there are often few observations in the past returns history large enough to fall in the set $C$. This fact prevents the use of commonly used statistical estimation methodologies which rely on the availability of considerable amounts of observations. The following result from extreme value theory helps us to overcome this problem.

If $G$ is an extreme value distribution as in (2) then (de Haan and Resnick (1977)) there exists a finite measure $\nu$ such that, for any Borel set $A$ of $[0, \infty]^{d} \backslash \mathbf{0}$ and for a scaling constant $c>0$,

$$
c \nu(c A)=\nu(A),
$$

provided that $A$ is bounded away from the origin. The measure $\nu$ is called the exponent measure. The scaling property of the exponent measure, given by (4), is very useful for our purpose. The method is explained below in detail but in summary is as follows. The set $C$, of which we want to estimate the probability, is typically far in the tail. This set will be transformed (by a standardization appropriate for extreme events) into a set $c A$ for some set $A \in \mathbb{R}^{d}$ and scaling constant $c$. Although $c A$ is in the far tail $A$ is not and it is rather closer to the center of the support of the distribution. So the probability of $A$ can be estimated from the data. An estimate of the probability of set $c A$ is then obtained from the one estimated for $A$ using the scaling property (4). Finally, the probability of $C$ is given by the estimate of the probability of set $c A$.

The transformation from $C$ to $c A$ is as follows. First $\mathbf{R}$ is transformed using the normalizing constants from (2) and the shape parameter from (3), into return vectors $\tilde{\mathbf{R}}$,

$$
\tilde{\mathbf{R}}:=\left(1+\gamma \frac{\mathbf{R}-\mathbf{b}}{\mathbf{a}}\right)^{1 / \gamma}
$$

The second step is to find the probability of events of the transformed vectors in terms of the measure $\nu$. We use a result from de Haan and Resnick (1977). Let $A$ be any Borel set of $[0, \infty]^{d} \backslash \mathbf{0}$ bounded away from the origin and such that $\nu(\partial A)=0$. For a given $k$ such that $0<k \leq n$, we have that

$$
\lim _{n \rightarrow \infty} \frac{n}{k} P(\tilde{\mathbf{R}} \in A)=\nu(A)
$$

as $n \rightarrow \infty, k \rightarrow \infty$ and $k / n \rightarrow 0$. The probability $P(\tilde{\mathbf{R}} \in A)$ above depends on $n$ because $\tilde{\mathbf{R}}$ 
uses $\mathbf{a}$ and $\mathbf{b}$ which depend on $n$ by (1). $k$ is the number of observations, depending upon $n$, which are large enough to be considered in the tail of the distribution. The question of how to choose $k$ for a given sample is addressed in the next section within the estimation methodology.

Using (6), the probability of having large joint returns in a set $C$, for some $L$, can be obtained by writing $C$ as a transformation of a measurable set $A \subseteq[0, \infty]^{d} \backslash \mathbf{0}$ as

$$
C=\mathbf{a} \frac{(c A)^{\gamma}-\mathbf{1}}{\gamma}+\mathbf{b} \quad \text { or } \quad c A=\left(\mathbf{1}+\gamma \frac{C-\mathbf{b}}{\mathbf{a}}\right)^{1 / \gamma}
$$

where $c$ is a positive scaling constant as in (4).

When the set $C$ corresponds to very large joint movements there are typically very few, if any, observations in this set. This scarcity of observations in $C$ translates to the same lack of observations in its transformed set $c A$ which invalidates the use of an empirical estimator for $\nu(c A)$. But the normalizing transformation (5) shifts the return vectors towards the point 1. Hence, if we impose the requirement that the point $\mathbf{1}$ belongs to the boundary of $A$ then the set $A$ will have enough observations to allow the estimation of $\nu(A)$. This justifies the introduction of the scaling constant $c$.

Finally, assuming that $\mathbf{1}$ is on the boundary of $A$, we have that the probability of having a large joint asset return in the set $C$ is

$$
\begin{array}{rlrl}
p & :=P(\mathbf{R} \in C) & \\
& =P\left(\mathbf{R} \in \mathbf{a} \frac{(c A)^{\gamma}-\mathbf{1}}{\gamma}+\mathbf{b}\right) \\
& =P(\tilde{\mathbf{R}} \in c A) & \\
& \approx \frac{k}{n} \nu(c A) & \text { as } n \rightarrow+\infty & {[\text { by }(6)]} \\
& =\frac{k}{n c} \nu(A) & {[\text { by }(4)] .}
\end{array}
$$

The scaling property (4) is the key for the estimation of the probability of losses possibly never observed before. Result (8) shows how to obtain the probability of events depending on transformations of large joint asset returns.

\section{Joint large movements: estimation methodology}

Although expression (8) gives the probability of a large joint asset return being observed in $C$, we do not know $c, k, A$ and $\nu$ but only $n$ (the sample size). Estimation methods for 
these unknowns are addressed in this section. First we see how the shape parameter may be estimated, then $a$ and $b$, the measure $\nu, c$ and $A$, and finally $k$. We close the section by investigating declustering.

We use order statistics defined from a given finite random sample of size $n$ of univariate asset returns, $R_{1}, R_{2}, \ldots, R_{n}$. The ordered sample returns are denoted as

$$
R_{(n)} \leq R_{(n-1)} \leq \ldots \leq R_{(1)}
$$

The random variable $R_{(k)}$ is called the $k$ th upper-order statistic.

\subsection{The shape parameter (tail index)}

Define the function,

$$
M_{r}(R):=\frac{1}{k} \sum_{i=1}^{k}\left(\log R_{(i)}-\log R_{(k+1)}\right)^{r} .
$$

for $r=1,2$. The so called moment estimator of the shape parameter $\gamma$ (Dekkers et al. (1989)), is given by

$$
\hat{\gamma}:=M_{1}(R)+1-\frac{1}{2}\left(1-\frac{M_{1}(R)^{2}}{M_{2}(R)}\right)^{-1} .
$$

This is a consistent estimator of the shape parameter. Under an additional technical condition (Dekkers et al. (1989)) we have that if $\gamma \geq 0$ then $\sqrt{k}(\hat{\gamma}-\gamma)$ has asymptotically a normal distribution with mean zero and variance $1+\gamma^{2}$. For the case $\gamma<0$ the distribution of the statistic $\hat{\gamma}$ has a more complex expression (see Dekkers et al. (1989)) but we will see in the empirical illustration that we do not need this case.

We use the moment estimator for the shape parameter to decide if heavy-tail analysis is appropriate because it can be used whether $\gamma>0$ or $^{3} \gamma \leq 0$. The moment estimator may be plotted as a function of the number of upper-order statistics $k$ used in the estimation. We use these plots in our empirical study ahead to support the appropriateness of heavy-tail analysis.

\subsection{The normalizing constants}

To estimate the normalizing constants $a$ and $b$, the parameters of the univariate extreme value distribution given in (3), we use estimators studied by Dekkers et al. (1989). Define the functions,

$$
\bar{t}=t \wedge 0, \quad \rho_{1}(t)=\frac{1}{1-\bar{t}} \quad \text { and } \quad \rho_{2}(t)=\frac{2}{(1-\bar{t})(1-2 \bar{t})},
$$

\footnotetext{
${ }^{3}$ This is not possible with the Hill estimator (Hill (1975)) which can only be used when $\gamma>0$.
} 
then estimators for $a$ and $b$ are

$$
\begin{aligned}
\hat{b} & :=R_{(k+1)} \\
\hat{a} & :=\frac{R_{(k+1)} \sqrt{3 M_{1}(R)^{2}-M_{2}(R)}}{\sqrt{3\left(\rho_{1}(\hat{\gamma})\right)^{2}-\rho_{2}(\hat{\gamma})}} .
\end{aligned}
$$

\subsection{The exponent measure}

In order to estimate the exponent measure $\nu$ we have to transform first the multivariate returns $\mathbf{R}_{1}, \mathbf{R}_{2}, \ldots, \mathbf{R}_{n}$ according to (5). However, as the real values of the normalizing constants and of the shape parameter are not known we have to use their estimators. Consequently what we can

calculate are actually estimates of the normalized returns which we denote by $\hat{\mathbf{R}}_{1}, \hat{\mathbf{R}}_{2}, \ldots, \hat{\mathbf{R}}_{n}$ obtained as

$$
\hat{\mathbf{R}}_{i}:=\left(\mathbf{1}+\hat{\gamma} \frac{\mathbf{R}_{i}-\hat{\mathbf{b}}}{\hat{\mathbf{a}}}\right)^{1 / \hat{\gamma}}
$$

for $i=1,2, \ldots, n$.

For the exponent measure $\nu$ we use the non-parametric estimator suggested by de Haan and Resnick (1993),

$$
\hat{\nu}_{n}(A):=\frac{1}{k} \sum_{i=1}^{n} \mathbb{I}\left(\hat{\mathbf{R}}_{i} \in A\right),
$$

where $\mathbb{I}$ denotes the indicator function. Recall that the tail of each marginal variable is assumed to have a parametric distribution given by (3). However, the estimation of the extremal dependence structure using (13) does not assume any particular parametric form. It becomes clear at this point why this approach to the estimation of large joint movements is semi-parametric.

\subsection{The scaling constant $c$ and the set $A$}

To calculate the probability of observing large joint movements in a set $C$, for some $L$, using (8) we need to estimate the scaling constant $c$ and the set $A$. We have to impose a condition in order to have $c$ and $A$ uniquely defined. We want $A$ to be such that we can use the nonparametric estimator $\hat{\nu}$. Hence, $A$ should contain transformed data observations $\hat{\mathbf{R}}$. That happens if we impose the requirement that the point $\mathbf{1}$ is on the boundary of $A$ (Dekkers et al. (1989)), given that $\hat{\mathbf{R}}$ are obtained using the standardization (12).

Given a set $C$, for some $L$, we can always define a function $f^{*}(\mathbf{R})$ such that

$$
C=\left\{\mathbf{R} \mid f^{*}(\mathbf{R}) \geq 1\right\}
$$


Each point $\mathbf{R}$ in the set of large losses can be written as the transform of a point $\hat{\mathbf{R}}$ by the inverse mapping of $(12)$,

$$
\mathbf{R}=\mathbf{a} \frac{\hat{\mathbf{R}}^{\gamma}-\mathbf{1}}{\gamma}+\mathbf{b}
$$

At this point we assume that the function $f$ is defined for the case when all returns take the same value. This assumption should not be too restrictive in practice. Hence, from the definition of the function $f^{*}$ there exists a value $x$ such that $\mathbf{R}=(x, x, \ldots, x)$ is solution of the equation $f^{*}(\mathbf{R})=1$ which is equivalent to the existence of a value $s$ such that $s .1$ is a solution of the equation

$$
f^{*}\left(\mathbf{a} \frac{(s \mathbf{1})^{\gamma}-\mathbf{1}}{\gamma}+\mathbf{b}\right)=1 .
$$

If we define $c$ as the solution of this equation, then the point $\mathbf{1}$ is on the boundary of $A$. Since we have only estimates of $\mathbf{a}, \mathbf{b}$ and $\boldsymbol{\gamma}$ we can only find an estimate $\hat{c}$ of $c$ as the solution of $f^{*}(\hat{\mathbf{a}}((s \mathbf{1}) \hat{\gamma}-\mathbf{1}) / \hat{\gamma}+\hat{\mathbf{b}})=1$.

Finally, from (7) we define

$$
\hat{A}:=\frac{1}{\hat{c}}\left(\mathbf{1}+\hat{\gamma} \frac{C-\hat{\mathbf{b}}}{\hat{\mathbf{a}}}\right)^{1 / \hat{\gamma}}
$$

and we use

$$
\hat{p}:=\frac{k}{n \hat{c}} \hat{\nu}_{n}(\hat{A})
$$

as the estimator of $P(\mathbf{R} \in C)$.

\subsection{The number of upper-order statistics $k$}

The choice of the number of upper-order statistics $k$ used in the analysis is important. If $k$ is small, which means that we use few upper-order statistics, then the parameter estimates obtained have a large variance. If we use many upper-order statistics, so $k$ is large, then the parameter estimates are biased.

We use a graphic technique from Stărică (1999) to choose $k$. The idea is to use the scaling property (4). If the number $k$ is correct then the plot of the scaling ratio $s \hat{\nu}(s \hat{A}) / \hat{\nu}(\hat{A})$ is approximately 1 when the scaling constant $s$ takes values around 1 . In practice we produce several plots of the scaling ratio as a function of $s$ for various values of $k$. Then we choose the $k$ that produces the plot closer to the horizontal line at height 1 for values of the scaling constant $s$ around 1. Figure (1) displays the plots for our data and for the chosen $k$. For details on the implementation of this method we refer to Stărică (1999) and Resnick (2006). 


\subsection{The declustering of extremes}

It is a common finding in finance that returns on prices are heteroscedastic and have volatility persistence. The data used in our study is not an exception. This phenomena causes serial dependence of extremes, and is a major problem since the estimation procedure assumes serial independence.

The first approach we use to overcome this problem is called the blocks method; see Section 8.1 of Embrechts et al. (1997). We divide the sample in blocks, in our case quarters, and select the maximum observation in each block. Using this procedure, we obtain quarterly maxima observations and model the probability of having a large loss in a quarter.

A second approach we use, concentrates on the clusters of volatility in order to obtain independent observations of maxima. This relies on the so-called peaks-over-threshold method; see Balkema and de Haan (1974) and Pickands (1975). The exceedances ${ }^{4}$ above a sufficiently high threshold follow a generalized Pareto distribution (GDP) and occur according to a homogeneous Poisson process. This implies that the times between successive events are independent and identically distributed with an exponential distribution. In the presence of volatility clusters the same result is valid for cluster maxima; see Section 8.1 of Embrechts et al. (1997). Hence, in our study we select the maximum observation in each cluster and perform the necessary specification tests.

\section{Joint large movements: empirical study}

We explore the extremal dependence structure among three major international equity markets using the stock indices FTSE 100, Nikkei 225 and S\&P 500. We evaluate a risk measure of these three indices by estimating the probability of having a major loss in a portfolio composed of them. The data consists of prices covering the period from April 1984 until March 2007. We use logarithmic daily returns obtained from closing prices. As it is common in the literature, we define the losses resulting from a drop in prices as positive and the gains as negative.

There is documented evidence that the US market has the greatest influence in the other stock markets; see for instance Martens and Poon (2001). From the set considered here, the US market is also the last to close every day. Hence, an extreme event in the US market

\footnotetext{
${ }^{4}$ The exceedances of a series of loss returns are the losses minus a chosen threshold.
} 
can be expected to have a major effect on the other two markets on the following day. For these reasons, we pair returns from FTSE 100 and Nikkei 225 from the same day with the S\&P 500 return from the previous day. Returns synchronization is more important when volatility models are to be used to filter the returns. It is less relevant in our case as we use declustering in order to obtain independent observations of maxima, but still we perform the synchronization.

\subsection{Descriptive statistics}

Descriptive statistics of the data can be found in Table 4 of Appendix A. This summarizes information about the unconditional distribution of the returns on the three indices. The statistics show that neither index returns had a significant trend over the period considered: the three sample means are very small relative to the corresponding standard deviation estimates. All series exhibit skewness to the losses as well as excess kurtosis. This indicates departure from unconditional univariate normality for these series. The unconditional linear correlation coefficient points to a strong linear relation between the three index returns, being stronger the relation between FTSE 100 and S\&P 500, and between Nikkei 225 and the S\&P 500, than the relation between FTSE 100 and Nikkei 225.

Concerning the temporal dynamics of the return series, we use the Ljung-Box test for serial correlation and the Lagrange Multiplier test from Engle (1982) for heteroscedasticity. For both returns and losses we reject the null hypothesis of no serial correlation for the three indices. These results are reported in Table 5 in Appendix A together with the statistics obtained for the heteroscedasticity test. From the results we reject the null hypothesis of no heteroscedasticity for the three indices.

\subsection{Univariate Extreme Value Analysis and Declustering}

The presence of serial correlation and heteroscedasticity indicates the existence of clusters of losses in the data. This is often the case in finance data and a common approach is to decluster the losses. The declustered losses are then independent observations fulfilling the conditions for extreme value analysis ${ }^{5}$. To decluster the losses our first approach is to select the maximum

\footnotetext{
${ }^{5}$ When one is interested in the conditional tail behavior, another possibility is to use a volatility filter. In this empirical study we concentrate on the unconditional tail.
} 
loss observed in each quarter. This procedure results in 92 observations of maxima for each index. Results of a serial correlation test on these data are given in Table 6 in Appendix B. The table shows we can assume that the observations are independent. We tested for serial correlation up to the fourth moment in the three indices obtaining p-values close to one in all cases. The Lagrange Multiplier statistics, reported in Table 6, reveal no heteroscedasticity in the 92 quarterly maxima observations.

In order to investigate if the quarterly maximum losses have heavy tails we estimate the shape parameter, $\gamma$ using the moments estimator given by (9), for each index. Figure 5 in Appendix $\mathrm{B}$ displays the plots of the moment estimates of $\gamma$ as a function of the number of upper-order statistics used in the estimation. The estimates are above zero but the $95 \%$ confidence intervals for $\gamma$ are not completely above zero. Nevertheless, in the absence of other evidence that $\gamma \leq 0$, we shall assume the most conservative case that the three indices have heavy tailed loss distributions. Still, we use the moments estimator for $\gamma$ and not the Hill estimator since the Hill estimator can be used only when $\gamma$ is definitely positive.

One can argue that choosing the maxima over quarters is not appropriate because periods of volatility clustering are not necessarily linked to calendar periods. In a second approach to declustering, instead of choosing the maxima over each quarter, we choose the maxima over each volatility cluster. That raises the question of how to define a volatility cluster in terms of large joint movements.

We assume that the losses of a volatility cluster are large enough to be considered in the tail of the distribution. To identify the volatility clusters we use the peaks-over-threshold method (see Embrechts et al. (1997) for details) to choose a threshold for each index above which the losses belong to the tail of the distribution. We assume that a volatility cluster is a set of excess losses in the tail with a time gap between consecutive losses of less than 5 days. The thresholds must be set high enough such that the maximum exceedances per cluster are independent generalized Pareto and the time gaps are independent exponentially distributed. Further, we consider that there is a volatility cluster in the portfolio only if there is a volatility cluster in at least one of the three indices composing the portfolio.

Implementing this declustering procedure produces 200 volatility cluster maximum losses. Table 7 in Appendix $\mathrm{C}$ displays the thresholds used and specification tests. The test of goodness of fit of the exceedances does not reject the null hypothesis that these follow a generalized Pareto 
distribution. The exceedances and the time gaps show no sign of serial correlation. Concerning the time gap distribution the QQ-plots, displayed in Figure 6 in Appendix C, do not show evidence against an exponential distribution.

Table 8 (Appendix D) shows the results of heteroscedasticity tests on the volatility cluster maximum losses. We conclude that there is no heteroscedasticity. As for the quarterly maximum losses, tests for serial correlation up to the fourth moment give indication of no serial dependence in the volatility cluster maximum losses.

We conclude that the volatility cluster maximum losses are independent. We compute and plot in Figure 7 the moment estimates of the shape parameter $\gamma$ as a function of the number of upper-order statistics used. For all three indices the estimates of $\gamma$ are above zero. Comparing these estimates with the ones plotted in Figure 5 for the quarterly maxima, we observe that for the volatility cluster maxima the $95 \%$ confidence intervals for $\gamma$ are even higher above zero. The assumption that the three index losses have heavy tails is strengthened by these results.

Before proceeding to the estimation of the portfolio tail probabilities we test for asymptotic dependence between the three indices using the same test as Poon et al. (2004). According to this test, non rejection of the hypothesis that the test statistic, $\bar{\chi}$, equals one indicates asymptotic dependence. Table 1 has the test statistics and standard errors for the three pairs of indices. Given the results obtained we cannot reject the hypothesis of asymptotic

\begin{tabular}{cccc}
\multicolumn{4}{c}{ Asymptotic dependence test } \\
\hline & FTSE-Nikkei & FTSE-S\&P & Nikkei-S\&P \\
\hline Quarterly maximum losses, $\bar{\chi}$ & 1.042 & 0.997 & 0.977 \\
& $(0.3151)$ & $(0.2913)$ & $(0.3015)$ \\
Vol. cluster maximum losses, $\bar{\chi}$ & 0.856 & 1.067 & 1.055 \\
& $(0.1925)$ & $(0.2109)$ & $(0.2269)$ \\
\hline
\end{tabular}

Table 1: Asymptotic dependence test statistic values for the three pairs between FTSE 100, Nikkei 225 and S\&P 500. In all cases the test statistic is not significantly different from one. Hence, the null hypothesis of asymptotic dependence cannot be rejected.

dependence. This test ensures that tail probabilities are not being overestimated when we use the semi-parametric estimator in the sequel.

\subsection{Computation of the portfolio tail probabilities}

From the declustering analysis 92 quarterly maximum losses and 200 volatility cluster maximum losses over the period April 1984 until March 2007 were obtained for each of the three indices 
Quarterly Max, k = 19

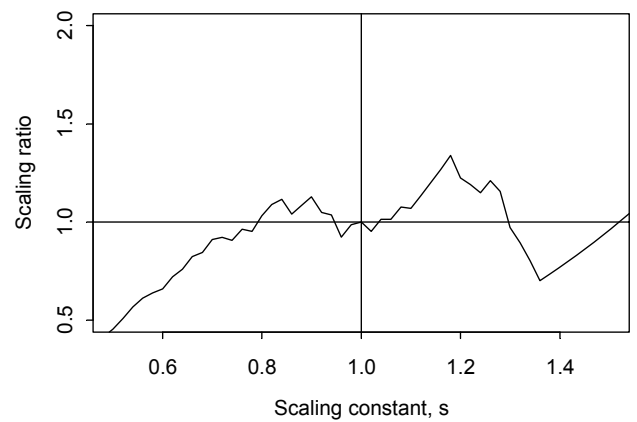

Vol.Cluster Max, $\mathrm{k}=53$

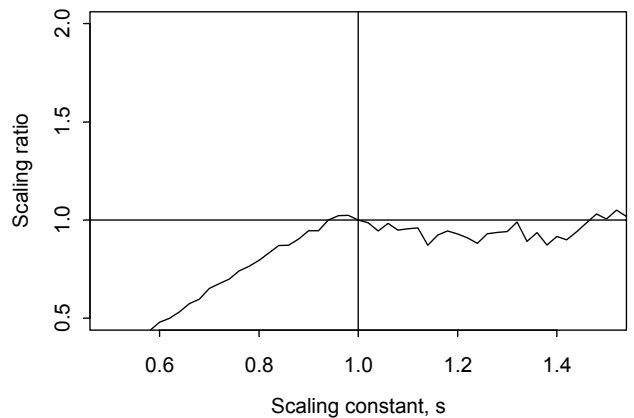

Figure 1: Stărică plots for the 92 quarterly maximum losses and 200 volatility cluster maximum losses of the three indices FTSE 100, Nikkei 225 and S\&P 500.

FTSE 100, Nikkei 225 and S\&P 500. From the Stărică plots in Figure 1, we choose to use $k=19$ upper-order statistics of the quarterly maximum losses and $k=53$ of the volatility cluster maximum losses. We made Stărică plots for various values of $k$ and choose the $k$ which produces the scaling ratio roughly equal to 1 in a neighborhood of 1 .

Once the number of upper-order statistics to be used is chosen we can estimate the parameters of the three univariate marginal extreme value distributions; $a_{i}, b_{i}$ and $\gamma_{i}$ for $i=1,2,3$. The corresponding estimates obtained with the moments estimator are reported in Table 2 .

Extreme value distribution parameter estimates

\begin{tabular}{|c|c|c|c|c|c|c|}
\hline \multirow[b]{2}{*}{ Parameter } & \multicolumn{3}{|c|}{ Quarterly maximum losses $(k=19)$} & \multicolumn{3}{|c|}{ Vol. cluster maximum losses $(k=53)$} \\
\hline & FTSE 100 & Nikkei 225 & $\mathrm{~S} \& \mathrm{P} 500$ & FTSE 100 & Nikkei 225 & S\&P 500 \\
\hline$\gamma$ & 0.4300 & 0.2364 & 0.4420 & 0.3671 & 0.1936 & 0.4533 \\
\hline & $(0.2497)$ & $(0.2357)$ & $(0.2508)$ & $(0.1449)$ & $(0.1386)$ & $(0.1494)$ \\
\hline$a$ & 0.0059 & 0.0135 & 0.0147 & 0.0058 & 0.0110 & 0.0080 \\
\hline$b$ & 0.0296 & 0.0432 & 0.0305 & 0.0199 & 0.0296 & 0.0192 \\
\hline
\end{tabular}

Table 2: Extreme value distribution parameter estimates and estimated asymptotic standard errors (in parentheses) for the maximum losses on FTSE 100, Nikkei 225 and S\&P 500.

We can now compute the probability of having a large loss in the portfolio composed of the FTSE 100, the Nikkei 225 and the S\&P 500. Let us consider first the case of estimating the probability of having a loss larger than $20 \%$ on an equally weighted portfolio. This means to estimate the probability of the occurrence of

$$
C=\left\{\left(R_{1}, R_{2}, R_{3}\right) \mid \frac{1}{3} R_{1}+\frac{1}{3} R_{2}+\frac{1}{3} R_{3} \geq 0.2\right\},
$$

where $R_{j}, j=1,2,3$ represent the (positive) return losses on FTSE 100, Nikkei 225 and S\&P 500 respectively. The estimates of the normalizing constants and shape parameter are 




Figure 2: Quarterly maximum losses on the three indices FTSE 100, Nikkei 225 and S\&P 500. The losses are normalized using (12) and the plot is in log-scale. The goal is to estimate the probability of having a loss in the region above the outer surface. This is obtained from the observations in the region above the inner surface using the scaling property (4).

now used to transform the data, using (12), into normalized maximum pseudo-observations. We plot in Figure 2 the normalized quarterly maximum losses for the three indices in log-scale. The region above the outer surface corresponds to $C$ (defined by (16)) transformed into $\hat{c} \hat{A}$ using (14). The estimate of the scaling constant is $\hat{c}=146.5595$ which produces $\hat{A}$ given by the region above the inner surface plotted in Figure 2. Using (15) we obtain an estimate for the probability of having a loss larger than $20 \%$ in a quarter of $\hat{p}=0.1335 \%$. For the same portfolio, the estimated probability of having a loss larger than $20 \%$ in a volatility cluster is $\hat{p}=0.05315 \%$ with $\hat{c}=451.5805$. We observe that the probability of a portfolio loss as large as $20 \%$ is still not close to zero. The volatility cluster probability is less than a half the quarterly probability. This result is consistent with the fact that we have 92 quarters but 200 volatility clusters during the period covered by the data.

Next we consider two portfolios with different component weights. For each portfolio we estimate the probability of occurrence of a loss larger than a given value for several possible values of large losses. This corresponds to estimate the survival function ${ }^{6}$ of the losses. Figure 3 displays the plots of the estimated survival functions of large portfolio losses. The solid line gives

\footnotetext{
${ }^{6}$ We use the usual definition where the survival function is one minus the distribution function.
} 

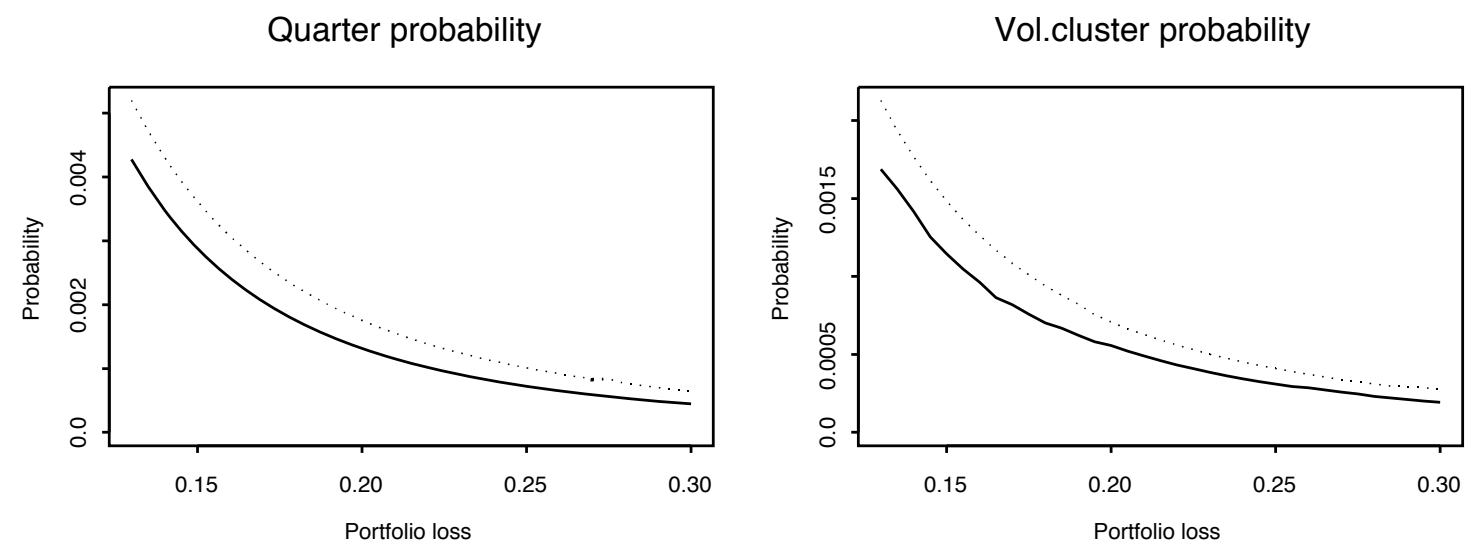

Figure 3: Plots of the quarterly and the volatility cluster probabilities ( $y$-axis) of a portfolio loss larger than a given value ( $x$-axis). The solid line is the plot for an equally weighted portfolio of the three indices FTSE 100, Nikkei 225 and S\&P 500. The dotted line is the plot of a portfolio with weights $25 \%, 25 \%$ and $50 \%$ respectively for the same indices.

the probabilities of an equally weighted portfolio and the doted line gives the probabilities for a portfolio with weights $25 \%, 25 \%$ and $50 \%$ of the indices FTSE 100, Nikkei 225 and S\&P 500 respectively. The plots show portfolio loss probabilities for quarterly maximum (left) and for the case of volatility cluster maximum (right). From these plots we observe that the weights of the portfolio have more effect on the probabilities of smaller large losses than of larger large losses since the two curves become closer when the value of the portfolio loss increases.

The effect of different weights in the loss probability deserves further investigation. For the quarterly maximum losses and for the cluster maximum losses we consider the probability of two fixed cases: a portfolio loss of $10 \%$ or more and a loss of $20 \%$ or more. For each case, we estimate the probabilities for portfolios with different weights of the three indices (we assume that the weights have to be positive and add up to one). Figure 4 displays the plots of the probability of a portfolio loss larger than $20 \%$ computed from the quarterly maximum losses (on the left) and from the cluster maximum losses (on the right). The probabilities are plotted as a function of the weights of FTSE 100 and Nikkei 225. The weights of S\&P 500 are determined from these.

We can observe from the plots that the estimated probabilities lie very close to an interpolating surface in both plots. This fact indicates stability of the estimation procedure. The probability still gets larger when the weight of the index S\&P 500 increases. The probability 



Figure 4: Estimates of the probability of observing a portfolio loss larger than $20 \%$ for different weights of the three indices FTSE 100, Nikkei 225 and S\&P 500. The left plot has the probabilities obtained from the quarterly maximum losses and the right plot has the cluster maximum probabilities. There is an interpolating surface indicated by a grid through the estimated probabilities in each plot.

decreases when the weight of the S\&P 500 index gets smaller for any combination of weights from the other two indices. Given that we choose a small weight from the S\&P 500 index there is no significant difference in the probability of a large loss between the weights given to each of the other two indices. To see this more clearly we list in Table 3 the estimated probabilities for a fixed relatively small weight, 10\%, the of S\&P 500 index and various combinations for the FTSE 100 and Nikkei 225 weights. It gives loss probabilities for losses of both 20\% (as in Figure 4) and in addition for $10 \%$. From the values listed in Table 3 we can see an increase

\begin{tabular}{cccccc}
\multicolumn{3}{c}{ Probabilities (in \%) of a large loss for different portfolio weights } \\
\cline { 1 - 3 } Portfolio weights & \multicolumn{2}{c}{ Quarterly maximum losses } & & \multicolumn{2}{c}{ Vol. cluster maximum losses } \\
\cline { 2 - 3 } \cline { 5 - 6 } (FTSE , Nikkei) & $20 \%$ & $10 \%$ & & $20 \%$ & $10 \%$ \\
\hline$(0.1,0.8)$ & 0.08474 & 0.99804 & & 0.02756 & 0.40553 \\
$(0.2,0.7)$ & 0.07515 & 0.78572 & & 0.03047 & 0.33855 \\
$(0.3,0.6)$ & 0.07607 & 0.65249 & & 0.03021 & 0.31366 \\
$(0.4,0.5)$ & 0.07692 & 0.61699 & & 0.02861 & 0.28533 \\
$(0.5,0.4)$ & 0.06910 & 0.58196 & & 0.02891 & 0.24869 \\
$(0.6,0.3)$ & 0.07439 & 0.48723 & & 0.02920 & 0.24343 \\
$(0.7,0.2)$ & 0.07138 & 0.46169 & & 0.03215 & 0.22841 \\
$(0.8,0.1)$ & 0.06865 & 0.43732 & & 0.03242 & 0.22930 \\
\hline
\end{tabular}

Table 3: Estimates (in \%) of the probability of observing a large portfolio loss for different weights of the indices FTSE 100 and Nikkei 225, given a fixed weight of $10 \%$ of the index $\mathrm{S} \& \mathrm{P}$ 500. The estimates are given for the quarter losses and for the volatility cluster losses.

in the probability of a large portfolio loss when the weight of the Nikkei 225 increases. This is more evident for a less extreme loss of $10 \%$ than for the larger extreme loss of $20 \%$. This 
is in agreement with what we observed in Figure 3. Still, the main conclusion is that extreme losses in a portfolio composed by the three indices increases substantially with the weight of the S\&P 500 index. Note from the results in Table 3 that the probability of a loss larger than $20 \%$ in a portfolio with $10 \%$ of S\&P 500 and $10 \%$ of FTSE 100 is $0.08474 \%$ per quarter and $0.02756 \%$ per volatility cluster. If we increase the weight of the index S\&P 500 to $80 \%$ then the probability of a loss larger than $20 \%$ increases to $0.28355 \%$ per quarter and to $0.10501 \%$ per volatility cluster. Hence, increasing the weight of the index S\&P 500 from $10 \%$ to $80 \%$ implies an increase of more than three times in the probability of a loss larger than $20 \%$ per quarter and per volatility cluster. The S\&P 500 has a much stronger effect on large losses than either of the other two indices.

We observe that the estimated tail index for the FTSE 100 index $(\hat{\gamma}=0.4300)^{7}$ is roughly similar to the estimated tail index for the S\&P $500(\hat{\gamma}=0.4420)$, and both are signicantly larger than the estimate for the Nikkei $225(\hat{\gamma}=0.2364)$. Although having similar univariate tail heaviness, we have noted that the S\&P 500 index has a much stronger effect on large portfolio losses than the FTSE 100. This shows the importance of modelling large co-movements and how the semi-parametric estimator allows us to understand the effect of each portfolio component in large portfolio losses.

\subsection{Applications and implications for finance practice}

The most obvious application of the semi-parametric methodology is in portfolio risk assessment, in particular, for computing portfolio Value-at-Risk (VaR) and expected shortfall. Being able to quantify the asymptotic dependence between the FTSE 100, the Nikkei 250 and the S\&P 500 makes it possible to reduce the portfolio extreme risk. In general, the method allows to explore the possibilities of tail diversification reducing the consequences of tail risk concentration during crisis.

In the estimation of portfolio tail measures, for VaR for instance, with parametric models it is necessary to use Monte Carlo simulation methods. Because the focus is in rare tail events, in practice it is necessary to reduce the amount of simulation by using more elaborate techniques such as importance sampling. The semi-parametric method presented here avoids the use of these computationally expensive methods.

\footnotetext{
${ }^{7}$ For quarterly maximum. The results obtained for the cluster maximum point in the same direction.
} 


\section{Conclusion}

We describe in this paper a methodology for estimating the probability of events depending on joint large movements of asset prices observed only a few times in the past history. The methodology uses a semi-parametric approach from extreme value theory. Applications of this estimation procedure include: the computation of the probability of large portfolio losses with implications for portfolio choice; estimation of joint credit defaults which is crucial for the valuation of credit derivatives; estimation of the tail dependence between a hedging instrument and the underlying asset; the valuation of options depending on joint large movements.

The method is particularly interesting for portfolio applications and questions involving multiname products because increasing the number of components does not make the computations more difficult. This overcomes the curse of dimensionality problem usual in multivariate problems.

In this paper we also stress the importance of the assumptions underlying the methodology. We describe a procedure for verifying these assumptions and check that they hold for our empirical example. The risk of overlooking this aspect is to overestimate probabilities of large joint movements and consequently, for instance, to overestimate measures of tail risk. One of the big advantages of the estimation method presented is that there is no need to specify any dependence structure in order to estimate the probability of large joint asset price movements.

In the empirical study we find a positive probability of occurrence of joint large movements of prices of FTSE 100, Nikkei 225 and S\&P 500. Each of the index returns is heavy tailed. Although having similar univariate tail heaviness, the S\&P 500 index has a much larger contribution to large portfolio losses than the FTSE 100. The higher the proportion of the S\&P 500 index the higher the probability of having an extreme loss in the portfolio. Furthermore, for a fixed weight of S\&P 500 there is no evidence of much difference between FTSE 100 and Nikkei 225 on large portfolio losses. 


\section{Appendix A. Descriptive statistics}

\begin{tabular}{|c|c|c|c|}
\hline \multicolumn{4}{|c|}{ Summary statistics } \\
\hline & FTSE 100 & Nikkei 225 & S\&P 500 \\
\hline Mean & 0.00029 & 0.00007 & 0.00037 \\
\hline Standard deviation & 0.01017 & 0.01373 & 0.01044 \\
\hline Skewness & 0.54641 & 0.12231 & 2.01278 \\
\hline Kurtosis & 8.11432 & 7.46611 & 44.72246 \\
\hline \multirow{3}{*}{ Linear correlation } & & Nikkei 225 & S\&P 500 \\
\hline & FTSE 100 & 0.26444 & 0.31467 \\
\hline & Nikkei 225 & 1 & 0.34295 \\
\hline
\end{tabular}

Table 4: Summary statistics for the stock market daily returns on FTSE 100, Nikkei 225 and S\&P 500 over the period April 1984 to March 2007.

Tests on the dynamics of the return series

\begin{tabular}{llccc}
\hline \multicolumn{5}{c}{ Tests on the dynamics of the return series } \\
\hline Serial correlation: returns & FTSE 100 & Nikkei 225 & S\&P 500 \\
& Pest statistic & 54.1227 & 32.3944 & 29.7475 \\
& & 0.0000 & 0.0012 & 0.0030 \\
Serial correlation: losses & Test statistic & 1469.9310 & 925.1983 & 859.1296 \\
& P-value & 0.0000 & 0.0000 & 0.0000 \\
& & & & \\
ARCH effects: returns & Test statistic & 1344.815 & 492.935 & 324.295 \\
& P-value & 0.000 & 0.000 & 0.000 \\
\hline
\end{tabular}

Table 5: Test statistics and p-values for the null hypotheses of no serial correlation (Ljung Box test with 12 lags) and no ARCH effects (Lagrange Multiplier test) of the stock market returns and losses on FTSE 100, Nikkei 225 and S\&P 500 over the period April 1984 until March 2007. 


\section{Appendix B. Declustering: quarterly maxima}

\begin{tabular}{|c|c|c|c|c|}
\hline & & FTSE 100 & Nikkei 225 & S\&P 500 \\
\hline \multirow[t]{2}{*}{ Serial correlation: LB test } & Test statistic & 7.9412 & 12.7297 & 9.5094 \\
\hline & $\mathrm{P}$-value & 0.7897 & 0.3890 & 0.6589 \\
\hline \multirow[t]{2}{*}{ ARCH effects: LM test } & Test statistic & 0.953 & 5.3012 & 0.7918 \\
\hline & P-value & 1.000 & 0.9472 & 1.000 \\
\hline
\end{tabular}

Table 6: Test statistics and p-values for the null hypotheses of no serial correlation (Ljung Box test with 12 lags) and no ARCH effects (Lagrange Multiplier test) of the stock market maximum quarterly losses on FTSE 100, Nikkei 225 and S\&P 500 over the period April 1984 until March 2007.
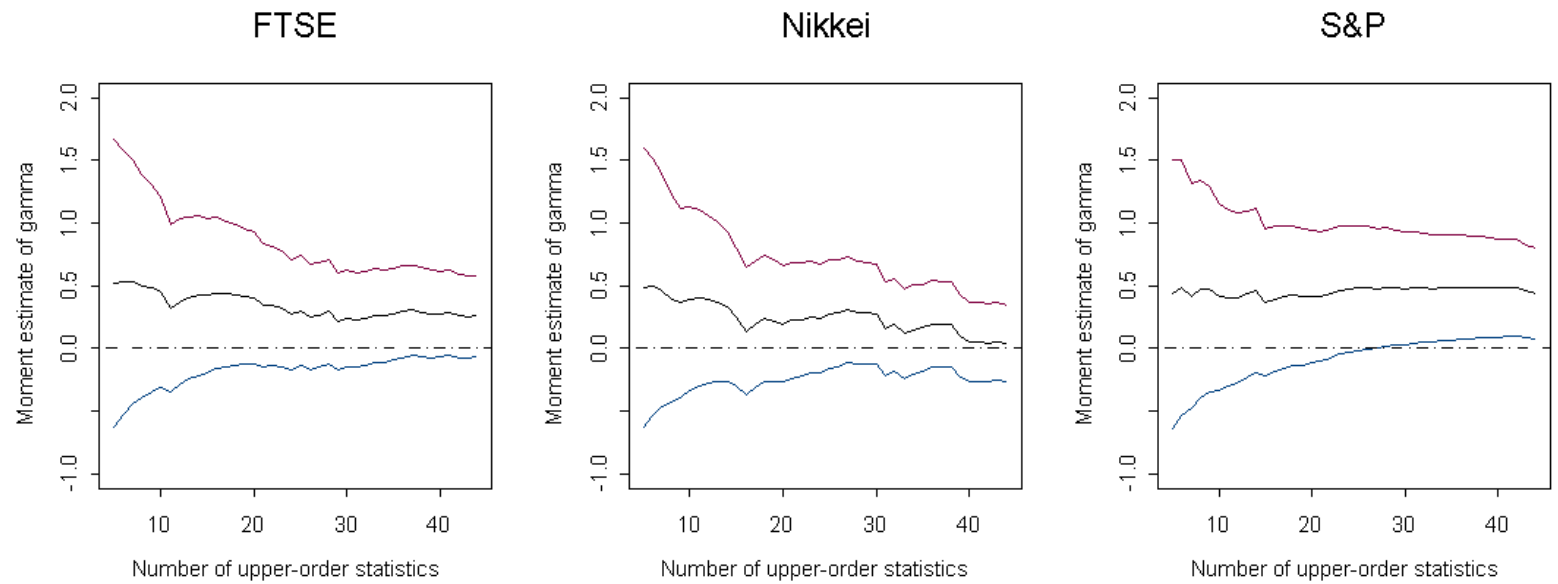

Figure 5: Moment estimates of the quarterly maximum losses extreme value parameter $\gamma$ as a function of the number of upper-order statistics used. The lower and upper lines are the limits of the $95 \%$ confidence interval for $\gamma$. 


\section{Appendix C. Declustering: volatility cluster maxima}

Thresholds and tests on the exceedances of the volatility cluster maximum losses

\begin{tabular}{|c|c|c|c|c|}
\hline & & FTSE 100 & $\mathrm{~S} \& \mathrm{P} 500$ & Nikkei 225 \\
\hline Threshold & & 0.013 & 0.0239 & 0.011 \\
\hline \multicolumn{5}{|c|}{ Exceedances } \\
\hline \multirow[t]{2}{*}{ Goodness-of-fit: GPD KS test } & Test statistic & 0.06464 & 0.04674 & 0.07234 \\
\hline & $\mathrm{P}$-value & 0.5 & 0.5 & 0.24237 \\
\hline \multirow[t]{2}{*}{ Serial correlation: LB test } & Test statistic & 9.51112 & 14.35754 & 10.29044 \\
\hline & $\mathrm{P}$-value & 0.98458 & 0.81191 & 0.98347 \\
\hline \multicolumn{5}{|c|}{ Time gaps } \\
\hline \multirow[t]{2}{*}{ Serial correlation: LB test } & Test statistic & 23.25398 & 11.69044 & 16.92291 \\
\hline & $\mathrm{P}$-value & 0.50484 & 0.98317 & 0.85193 \\
\hline
\end{tabular}

Table 7: Thresholds used to define the volatility clusters on FTSE 100, Nikkei 225 and S\&P 500. Test statistics and p-values for the generalized Pareto distribution goodness-of-fit (KolmogorovSmirnov test) and serial correlation (Ljung Box test) of the exceedances, and serial correlation of the time gaps between the volatility cluster maxima.
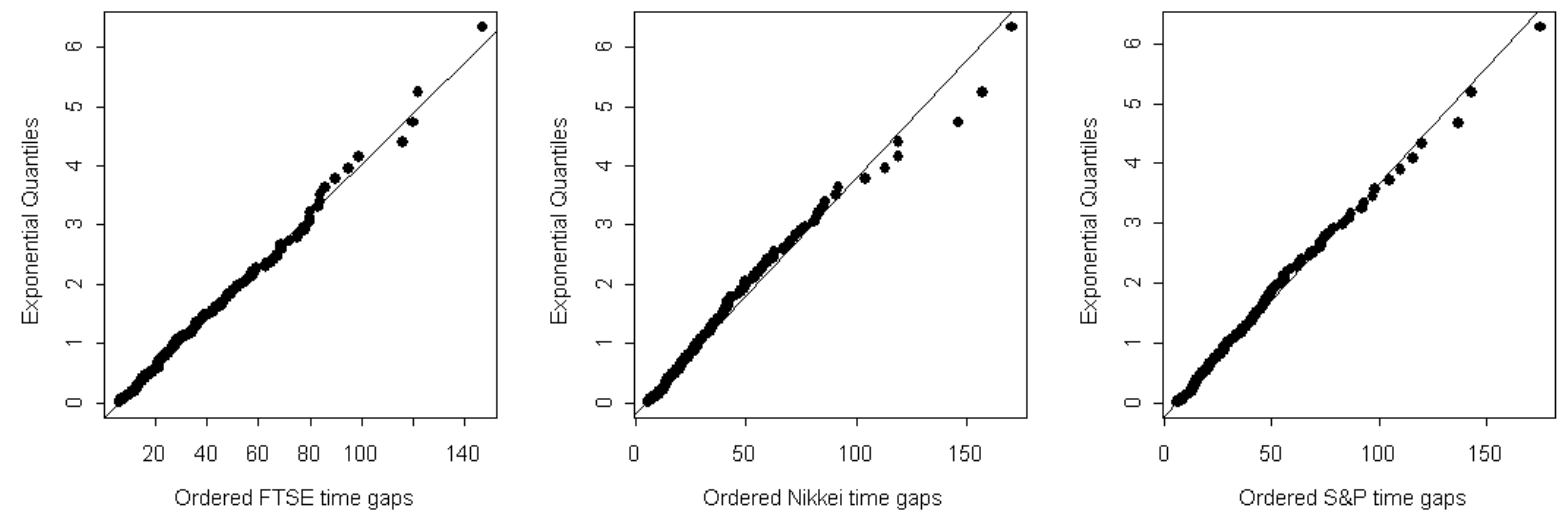

Figure 6: Exponential QQ-plots of the volatility cluster maximum losses time gaps on FTSE 100, Nikkei 225 and S\&P 500. 
Tests on the dynamics of the volatility cluster maximum losses

\begin{tabular}{llccc}
\hline & & FTSE 100 & S\&P 500 & Nikkei 225 \\
\hline \multirow{2}{*}{ ARCH effects: LM test } & Test statistic & 0.7225 & 2.8017 & 0.3903 \\
& P-value & 1.000 & 0.9968 & 1.000 \\
\hline
\end{tabular}

Table 8: Test statistics and p-values for the null hypotheses of no ARCH effects (Lagrange Multiplier test) of the stock market volatility cluster maximum losses on FTSE 100, Nikkei 225 and S\&P 500.

FTSE



Nikkei

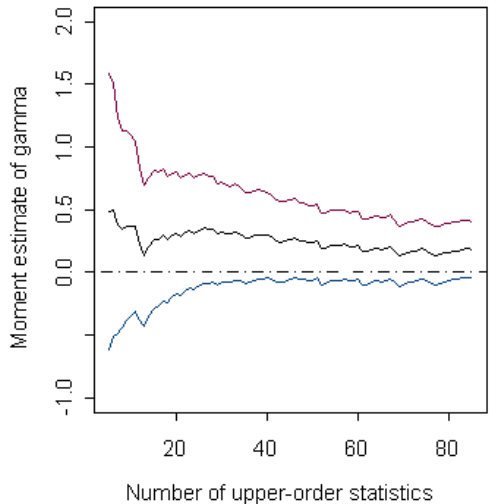

S\&P

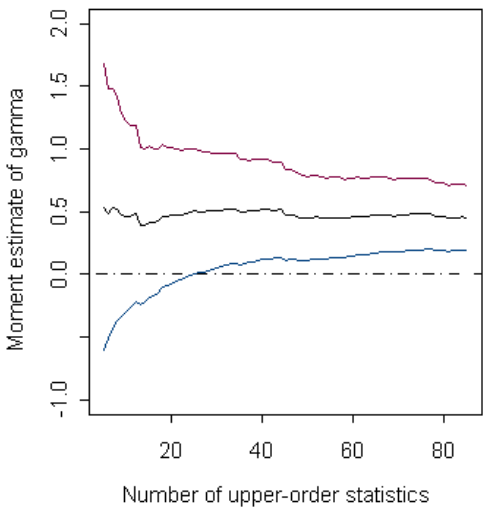

Figure 7: The plots display the moment estimates of the volatility cluster maximum losses extreme value parameter $\gamma$ as a function of the number of upper-order statistics used. The lower and upper lines are the limits of the $95 \%$ confidence interval for $\gamma$. 


\section{References}

Balkema, A. A. and de Haan, L. (1974). Residual life time at great age. The Annals of Probability, 2:792-804.

Cotter, J. (2006). Extreme value estimation of boom and crash statistics. The European Journal of Finance, 12(6-7):553-566.

Danielsson, J. and de Vries, C. G. (2000). Value at risk and extreme values. Annales D'Economie et de Statistique, 60:239-270.

de Haan, L. and de Ronde, J. (1998). Sea and wind: Multivariate extremes at work. Extremes, $1: 7-45$.

de Haan, L. and Resnick, S. (1977). Limit theory for multivariate extremes. Z. Wahrsch. Verw. Gebiete, 40:317-337.

de Haan, L. and Resnick, S. (1993). Estimating the limit distribution of multivariate extremes. Communications in Statistics. Stochastic Models, 9(2):275-309.

Dekkers, A. L. M., Einmahl, J. H. J., and de Haan, L. (1989). A moment estimator for the index of an extreme-value distribution. The Annals of Statistics, 17(4):1833-1855.

Diebold, F. X., Schuermann, T., and Stroughair, J. D. (1998). Pitfalls and opportunities in the use of extreme value theory in risk management. In: Advances in Computational Finance (Ed. A.-P.N. Refers, J.D. Moody and A.N. Burgess), Kluwer, Academic Press, Boston.

Embrechts, P., Klüppelberg, C., and Mikosch, T. (1997). Modelling Extremal Events for Insurance and Finance. Springer-Verlag, Berlin.

Engle, R. F. (1982). Autoregressive conditional heteroscedasticity with estimates of the variance of United Kingdom inflation. Econometrica, 50(4):987-1007.

Gumbel, E. J. (1961). Multivariate extremal distributions. Bulletin de l'Institut International de Statistiques, Session 33, Book 2, Paris.

Hartmann, P., Straetmans, S., and de Vries, C. G. (2001). Asset market linkages in crisis periods. Working paper, no. 71, European Central Bank, Frankfurt. 
Hill, B. M. (1975). A simple general approach to inference about the tail of a distribution. The Annals of Statistics, 3(5):1163-1174.

Jansen, D. W. and de Vries, C. G. (1991). On the frequency of large stock returns: putting booms and busts into perspectives. The review of economic and statistics, 73:18-24.

Longin, F. (1996). The asymptotic distribution of extreme stock markets returns. Journal of Business, 63:383-408.

Longin, F. (2005). The choice of the distribution of asset returns: How extreme value theory can help? Journal of Banking \& Finance, 29:1017-1035.

Longin, F. and Solnik, B. (2001). Extreme correlation of international equity markets. Journal of Finance, LVI(2):649-676.

Martens, M. and Poon, S. (2001). Returns synchronization and daily correlation dynamics between international stock markets. Journal of Banking and Finance, 25:1805-1827.

McNeil, A. and Frey, R. (2000). Estimation of tail-related risk measures for heteroscedastic financial time series: an extreme value approach. Journal of empirical finance, 7:271-300.

Pickands, J. (1975). Statistical inference using extreme order statistics. 3:119-131.

Poon, S. H., Rockinger, M., and Tawn, J. (2004). Extreme value dependence in financial markets: Diagnostics, models, and financial implications. The Review of Financial Studies, $17(2): 581-610$.

Resnick, S. (2006). Heavy-Tail Phenomena: Probabilistic and Statistical Modeling. SpringerVerlag, New York.

Stărică, C. (1999). Multivariate extremes for models with constant conditional correlations. J. Empirical Finance, 6:515-553. 


\section{List of other working papers:}

\section{8}

1. Roman Kozhan and Rozalia Pal, Firms' Investment under Financial Constraints: A Euro Area Investigation, WP08-07

2. Roman Kozhan and Mark Salmon, On Uncertainty, Market Timing and the Predictability of Tick by Tick Exchange Rates, WP08-06

3. Roman Kozhan and Mark Salmon, Uncertainty Aversion in a Heterogeneous Agent Model of Foreign Exchange Rate Formation, WP08-05

4. Roman Kozhan, Non-Additive Anonymous Games, WP08-04

5. Thomas Lux, Stochastic Behavioral Asset Pricing Models and the Stylized Facts, WP08-03

6. Reiner Franke, A Short Note on the Problematic Concept of Excess Demand in Asset Pricing Models with Mean-Variance Optimization, WP08-02

7. Alexandra Dias, Semi-parametric estimation of joint large movements of risky assets, WP08-01

\section{7}

1. Timur Yusupov and Thomas Lux, The Efficient Market Hypothesis through the Eyes of an Artificial Technical Analyst: An Application of a New Chartist Methodology to High-Frequency Stock Market Data, WP07-13

2. Liu Ruipeng, Di Matteo and Thomas Lux, True and Apparent Scaling: The Proximity of the Markov- Switching Multifractal Model to Long-Range Dependence, WP07-12

3. Thomas Lux, Rational Forecasts or Social Opinion Dynamics? Identification of Interaction Effects in a Business Climate Survey, WP07-11

4. Thomas Lux, Collective Opinion Formation in a Business Climate Survey, WP07-10

5. Thomas Lux, Application of Statistical Physics in Finance and Economics, WP07-09

6. Reiner Franke, A Prototype Model of Speculative Dynamics With Position-Based Trading, WP07-08

7. Reiner Franke, Estimation of a Microfounded Herding Model On German Survey Expectations, WP07-07

8. Cees Diks and Pietro Dindo, Informational differences and learning in an asset market with boundedly rational agents, WP07-06

9. Markus Demary, Who Do Currency Transaction Taxes Harm More: Short-Term Speculators or Long-Term Investors?, WP07-05

10. Markus Demary, A Heterogenous Agents Model Usable for the Analysis of Currency Transaction Taxes, WP07-04

11. Mikhail Anufriev and Pietro Dindo, Equilibrium Return and Agents' Survival in a Multiperiod Asset Market: Analytic Support of a Simulation Model, WP07-03

12. Simone Alfarano and Michael Milakovic, Should Network Structure Matter in Agent-Based Finance?, WP07-02

13. Simone Alfarano and Reiner Franke, A Simple Asymmetric Herding Model to Distinguish Between Stock and Foreign Exchange Markets, WP07-01

\section{6}

1. Roman Kozhan, Multiple Priors and No-Transaction Region, WP06-24

2. Martin Ellison, Lucio Sarno and Jouko Vilmunen, Caution and Activism? Monetary Policy Strategies in an Open Economy, WP06-23

3. Matteo Marsili and Giacomo Raffaelli, Risk bubbles and market instability, WP06-22

4. Mark Salmon and Christoph Schleicher, Pricing Multivariate Currency Options with Copulas, WP06-21 
5. Thomas Lux and Taisei Kaizoji, Forecasting Volatility and Volume in the Tokyo Stock Market: Long Memory, Fractality and Regime Switching, WP06-20

6. Thomas Lux, The Markov-Switching Multifractal Model of Asset Returns: GMM Estimation and Linear Forecasting of Volatility, WP06-19

7. Peter Heemeijer, Cars Hommes, Joep Sonnemans and Jan Tuinstra, Price Stability and Volatility in Markets with Positive and Negative Expectations Feedback: An Experimental Investigation, WP06-18

8. Giacomo Raffaelli and Matteo Marsili, Dynamic instability in a phenomenological model of correlated assets, WP06-17

9. Ginestra Bianconi and Matteo Marsili, Effects of degree correlations on the loop structure of scale free networks, WP06-16

10. Pietro Dindo and Jan Tuinstra, A Behavioral Model for Participation Games with Negative Feedback, WP06-15

11. Ceek Diks and Florian Wagener, A weak bifucation theory for discrete time stochastic dynamical systems, WP06-14

12. Markus Demary, Transaction Taxes, Traders' Behavior and Exchange Rate Risks, WP06-13

13. Andrea De Martino and Matteo Marsili, Statistical mechanics of socio-economic systems with heterogeneous agents, WP06-12

14. William Brock, Cars Hommes and Florian Wagener, More hedging instruments may destabilize markets, WP06-11

15. Ginwestra Bianconi and Roberto Mulet, On the flexibility of complex systems, WP06-10

16. Ginwestra Bianconi and Matteo Marsili, Effect of degree correlations on the loop structure of scale-free networks, WP06-09

17. Ginwestra Bianconi, Tobias Galla and Matteo Marsili, Effects of Tobin Taxes in Minority Game Markets, WP06-08

18. Ginwestra Bianconi, Andrea De Martino, Felipe Ferreira and Matteo Marsili, Multi-asset minority games, WP06-07

19. Ba Chu, John Knight and Stephen Satchell, Optimal Investment and Asymmetric Risk for a Large Portfolio: A Large Deviations Approach, WP06-06

20. Ba Chu and Soosung Hwang, The Asymptotic Properties of AR(1) Process with the Occasionally Changing AR Coefficient, WP06-05

21. Ba Chu and Soosung Hwang, An Asymptotics of Stationary and Nonstationary AR(1) Processes with Multiple Structural Breaks in Mean, WP06-04

22. Ba Chu, Optimal Long Term Investment in a Jump Diffusion Setting: A Large Deviation Approach, WP06-03

23. Mikhail Anufriev and Gulio Bottazzi, Price and Wealth Dynamics in a Speculative Market with Generic Procedurally Rational Traders, WP06-02

24. Simonae Alfarano, Thomas Lux and Florian Wagner, Empirical Validation of Stochastic Models of Interacting Agents: A "Maximally Skewed" Noise Trader Model?, WP06-01

\section{5}

1. Shaun Bond and Soosung Hwang, Smoothing, Nonsynchronous Appraisal and CrossSectional Aggreagation in Real Estate Price Indices, WP05-17

2. Mark Salmon, Gordon Gemmill and Soosung Hwang, Performance Measurement with Loss Aversion, WP05-16

3. Philippe Curty and Matteo Marsili, Phase coexistence in a forecasting game, WP05-15

4. Matthew Hurd, Mark Salmon and Christoph Schleicher, Using Copulas to Construct Bivariate Foreign Exchange Distributions with an Application to the Sterling Exchange Rate Index (Revised), WP05-14

5. Lucio Sarno, Daniel Thornton and Giorgio Valente, The Empirical Failure of the Expectations Hypothesis of the Term Structure of Bond Yields, WP05-13

6. Lucio Sarno, Ashoka Mody and Mark Taylor, A Cross-Country Financial Accelorator: Evidence from North America and Europe, WP05-12

7. Lucio Sarno, Towards a Solution to the Puzzles in Exchange Rate Economics: Where Do We Stand?, WP05-11

8. James Hodder and Jens Carsten Jackwerth, Incentive Contracts and Hedge Fund Management, WP05-10

9. James Hodder and Jens Carsten Jackwerth, Employee Stock Options: Much More Valuable Than You Thought, WP05-09 
10. Gordon Gemmill, Soosung Hwang and Mark Salmon, Performance Measurement with Loss Aversion, WP05-08

11. George Constantinides, Jens Carsten Jackwerth and Stylianos Perrakis, Mispricing of S\&P 500 Index Options, WP05-07

12. Elisa Luciano and Wim Schoutens, A Multivariate Jump-Driven Financial Asset Model, WP0506

13. Cees Diks and Florian Wagener, Equivalence and bifurcations of finite order stochastic processes, WP05-05

14. Devraj Basu and Alexander Stremme, CAY Revisited: Can Optimal Scaling Resurrect the (C)CAPM?, WP05-04

15. Ginwestra Bianconi and Matteo Marsili, Emergence of large cliques in random scale-free networks, WP05-03

16. Simone Alfarano, Thomas Lux and Friedrich Wagner, Time-Variation of Higher Moments in a Financial Market with Heterogeneous Agents: An Analytical Approach, WP05-02

17. Abhay Abhayankar, Devraj Basu and Alexander Stremme, Portfolio Efficiency and Discount Factor Bounds with Conditioning Information: A Unified Approach, WP05-01

\section{4}

1. Xiaohong Chen, Yanqin Fan and Andrew Patton, Simple Tests for Models of Dependence Between Multiple Financial Time Series, with Applications to U.S. Equity Returns and Exchange Rates, WP04-19

2. Valentina Corradi and Walter Distaso, Testing for One-Factor Models versus Stochastic Volatility Models, WP04-18

3. Valentina Corradi and Walter Distaso, Estimating and Testing Sochastic Volatility Models using Realized Measures, WP04-17

4. Valentina Corradi and Norman Swanson, Predictive Density Accuracy Tests, WP04-16

5. Roel Oomen, Properties of Bias Corrected Realized Variance Under Alternative Sampling Schemes, WP04-15

6. Roel Oomen, Properties of Realized Variance for a Pure Jump Process: Calendar Time Sampling versus Business Time Sampling, WP04-14

7. Richard Clarida, Lucio Sarno, Mark Taylor and Giorgio Valente, The Role of Asymmetries and Regime Shifts in the Term Structure of Interest Rates, WP04-13

8. Lucio Sarno, Daniel Thornton and Giorgio Valente, Federal Funds Rate Prediction, WP04-12

9. Lucio Sarno and Giorgio Valente, Modeling and Forecasting Stock Returns: Exploiting the Futures Market, Regime Shifts and International Spillovers, WP04-11

10. Lucio Sarno and Giorgio Valente, Empirical Exchange Rate Models and Currency Risk: Some Evidence from Density Forecasts, WP04-10

11. Ilias Tsiakas, Periodic Stochastic Volatility and Fat Tails, WP04-09

12. Ilias Tsiakas, Is Seasonal Heteroscedasticity Real? An International Perspective, WP04-08

13. Damin Challet, Andrea De Martino, Matteo Marsili and Isaac Castillo, Minority games with finite score memory, WP04-07

14. Basel Awartani, Valentina Corradi and Walter Distaso, Testing and Modelling Market Microstructure Effects with an Application to the Dow Jones Industrial Average, WP04-06

15. Andrew Patton and Allan Timmermann, Properties of Optimal Forecasts under Asymmetric Loss and Nonlinearity, WP04-05

16. Andrew Patton, Modelling Asymmetric Exchange Rate Dependence, WP04-04

17. Alessio Sancetta, Decoupling and Convergence to Independence with Applications to Functional Limit Theorems, WP04-03

18. Alessio Sancetta, Copula Based Monte Carlo Integration in Financial Problems, WP04-02

19. Abhay Abhayankar, Lucio Sarno and Giorgio Valente, Exchange Rates and Fundamentals: Evidence on the Economic Value of Predictability, WP04-01

\section{2}

1. Paolo Zaffaroni, Gaussian inference on Certain Long-Range Dependent Volatility Models, WP02-12

2. Paolo Zaffaroni, Aggregation and Memory of Models of Changing Volatility, WP02-11

3. Jerry Coakley, Ana-Maria Fuertes and Andrew Wood, Reinterpreting the Real Exchange Rate - Yield Diffential Nexus, WP02-10 
4. Gordon Gemmill and Dylan Thomas, Noise Training, Costly Arbitrage and Asset Prices: evidence from closed-end funds, WP02-09

5. Gordon Gemmill, Testing Merton's Model for Credit Spreads on Zero-Coupon Bonds, WP0208

6. George Christodoulakis and Steve Satchell, On th Evolution of Global Style Factors in the MSCI Universe of Assets, WP02-07

7. George Christodoulakis, Sharp Style Analysis in the MSCI Sector Portfolios: A Monte Caro Integration Approach, WP02-06

8. George Christodoulakis, Generating Composite Volatility Forecasts with Random Factor Betas, WP02-05

9. Claudia Riveiro and Nick Webber, Valuing Path Dependent Options in the Variance-Gamma Model by Monte Carlo with a Gamma Bridge, WP02-04

10. Christian Pedersen and Soosung Hwang, On Empirical Risk Measurement with Asymmetric Returns Data, WP02-03

11. Roy Batchelor and Ismail Orgakcioglu, Event-related GARCH: the impact of stock dividends in Turkey, WP02-02

12. George Albanis and Roy Batchelor, Combining Heterogeneous Classifiers for Stock Selection, WP02-01

\section{1}

1. Soosung Hwang and Steve Satchell, GARCH Model with Cross-sectional Volatility; GARCHX Models, WP01-16

2. Soosung Hwang and Steve Satchell, Tracking Error: Ex-Ante versus Ex-Post Measures, WP01-15

3. Soosung Hwang and Steve Satchell, The Asset Allocation Decision in a Loss Aversion World, WP01-14

4. Soosung Hwang and Mark Salmon, An Analysis of Performance Measures Using Copulae, WP01-13

5. Soosung Hwang and Mark Salmon, A New Measure of Herding and Empirical Evidence, WP01-12

6. Richard Lewin and Steve Satchell, The Derivation of New Model of Equity Duration, WP0111

7. Massimiliano Marcellino and Mark Salmon, Robust Decision Theory and the Lucas Critique, WP01-10

8. Jerry Coakley, Ana-Maria Fuertes and Maria-Teresa Perez, Numerical Issues in Threshold Autoregressive Modelling of Time Series, WP01-09

9. Jerry Coakley, Ana-Maria Fuertes and Ron Smith, Small Sample Properties of Panel Timeseries Estimators with I(1) Errors, WP01-08

10. Jerry Coakley and Ana-Maria Fuertes, The Felsdtein-Horioka Puzzle is Not as Bad as You Think, WP01-07

11. Jerry Coakley and Ana-Maria Fuertes, Rethinking the Forward Premium Puzzle in a Nonlinear Framework, WP01-06

12. George Christodoulakis, Co-Volatility and Correlation Clustering: A Multivariate Correlated ARCH Framework, WP01-05

13. Frank Critchley, Paul Marriott and Mark Salmon, On Preferred Point Geometry in Statistics, WP01-04

14. Eric Bouyé and Nicolas Gaussel and Mark Salmon, Investigating Dynamic Dependence Using Copulae, WP01-03

15. Eric Bouyé, Multivariate Extremes at Work for Portfolio Risk Measurement, WP01-02

16. Erick Bouyé, Vado Durrleman, Ashkan Nikeghbali, Gael Riboulet and Thierry Roncalli, Copulas: an Open Field for Risk Management, WP01-01

\section{0}

1. Soosung Hwang and Steve Satchell, Valuing Information Using Utility Functions, WP00-06

2. Soosung Hwang, Properties of Cross-sectional Volatility, WP00-05

3. Soosung Hwang and Steve Satchell, Calculating the Miss-specification in Beta from Using a Proxy for the Market Portfolio, WP00-04

4. Laun Middleton and Stephen Satchell, Deriving the APT when the Number of Factors is Unknown, WP00-03 
5. George A. Christodoulakis and Steve Satchell, Evolving Systems of Financial Returns: AutoRegressive Conditional Beta, WP00-02

6. Christian S. Pedersen and Stephen Satchell, Evaluating the Performance of Nearest Neighbour Algorithms when Forecasting US Industry Returns, WP00-01

\section{9}

1. Yin-Wong Cheung, Menzie Chinn and Ian Marsh, How do UK-Based Foreign Exchange Dealers Think Their Market Operates?, WP99-21

2. Soosung Hwang, John Knight and Stephen Satchell, Forecasting Volatility using LINEX Loss Functions, WP99-20

3. Soosung Hwang and Steve Satchell, Improved Testing for the Efficiency of Asset Pricing Theories in Linear Factor Models, WP99-19

4. Soosung Hwang and Stephen Satchell, The Disappearance of Style in the US Equity Market, WP99-18

5. Soosung Hwang and Stephen Satchell, Modelling Emerging Market Risk Premia Using Higher Moments, WP99-17

6. Soosung Hwang and Stephen Satchell, Market Risk and the Concept of Fundamental Volatility: Measuring Volatility Across Asset and Derivative Markets and Testing for the Impact of Derivatives Markets on Financial Markets, WP99-16

7. Soosung Hwang, The Effects of Systematic Sampling and Temporal Aggregation on Discrete Time Long Memory Processes and their Finite Sample Properties, WP99-15

8. Ronald MacDonald and Ian Marsh, Currency Spillovers and Tri-Polarity: a Simultaneous Model of the US Dollar, German Mark and Japanese Yen, WP99-14

9. Robert Hillman, Forecasting Inflation with a Non-linear Output Gap Model, WP99-13

10. Robert Hillman and Mark Salmon, From Market Micro-structure to Macro Fundamentals: is there Predictability in the Dollar-Deutsche Mark Exchange Rate?, WP99-12

11. Renzo Avesani, Giampiero Gallo and Mark Salmon, On the Evolution of Credibility and Flexible Exchange Rate Target Zones, WP99-11

12. Paul Marriott and Mark Salmon, An Introduction to Differential Geometry in Econometrics, WP99-10

13. Mark Dixon, Anthony Ledford and Paul Marriott, Finite Sample Inference for Extreme Value Distributions, WP99-09

14. Ian Marsh and David Power, A Panel-Based Investigation into the Relationship Between Stock Prices and Dividends, WP99-08

15. Ian Marsh, An Analysis of the Performance of European Foreign Exchange Forecasters, WP99-07

16. Frank Critchley, Paul Marriott and Mark Salmon, An Elementary Account of Amari's Expected Geometry, WP99-06

17. Demos Tambakis and Anne-Sophie Van Royen, Bootstrap Predictability of Daily Exchange Rates in ARMA Models, WP99-05

18. Christopher Neely and Paul Weller, Technical Analysis and Central Bank Intervention, WP9904

19. Christopher Neely and Paul Weller, Predictability in International Asset Returns: A Reexamination, WP99-03

20. Christopher Neely and Paul Weller, Intraday Technical Trading in the Foreign Exchange Market, WP99-02

21. Anthony Hall, Soosung Hwang and Stephen Satchell, Using Bayesian Variable Selection Methods to Choose Style Factors in Global Stock Return Models, WP99-01

\section{8}

1. Soosung Hwang and Stephen Satchell, Implied Volatility Forecasting: A Compaison of Different Procedures Including Fractionally Integrated Models with Applications to UK Equity Options, WP98-05

2. Roy Batchelor and David Peel, Rationality Testing under Asymmetric Loss, WP98-04

3. Roy Batchelor, Forecasting T-Bill Yields: Accuracy versus Profitability, WP98-03

4. Adam Kurpiel and Thierry Roncalli, Option Hedging with Stochastic Volatility, WP98-02

5. Adam Kurpiel and Thierry Roncalli, Hopscotch Methods for Two State Financial Models, WP98-01 